\title{
Correlative Electron Microscopy and Chemical Imaging of Mycorrhizospheric Biofilms - a Capability Development
}

\author{
Alice Dohnalkova ${ }^{*}$, Zsuzsanna Balogh-Brunstad ${ }^{* *}$ and Kent Keller ${ }^{* * *}$ \\ *Environmental Molecular Sciences Laboratory, Pacific Northwest National Laboratory, \\ Richland, WA \\ ${ }^{* *}$ Geology \& Environmental Sciences, Hartwick College, Oneonta, NY \\ ${ }^{* * *}$ School of Earth and Environmental Sciences, Washington State University, Pullman, \\ WA
}

The formation of soils from rocks by weathering is the foundation of terrestrial ecosystems, and over geological timescale it represents a major feedback on atmospheric $\mathrm{CO}_{2}$ levels and therefore on the Earth's climate. Our understanding of rock weathering by aqueous dissolution reactions also integrates the significant presence of plants rootassociated fungi (mycorrhizal networks) and the unambiguous role of bacterial population associated with the hyphae emanating from mycorrhizal roots. This system, fuelled by plant photosynthesis, creates huge underground surface area for bacterial colonization. In return, mycorrhizal networks extract and transfer essential nutrients from soil particles to the host plant roots, degrade minerals and form the soils.

To study plant-driven weathering mechanisms, an experimental setup of column-type mycorrhizal seedling growth experiments will be used [1]. Varying degrees of nutrient limitations will be evaluated at the root system-mineral interface in terms of microbiology and mineral chemistry. As a part of the microscopy approach, a dependable scheme for representing the closest-to natural conditions needed to be developed. Given the hyphae microscopic dimensions (only 5um wide but up to $1 \mathrm{~mm}$ long) and other challenging characteristics such as strong adhesion to the minerals substrates further complicates the study of the weathering activity in the laboratory. Before the long-term laboratory column experiments was carried out, a test of capability was performed to establish protocols for morphological and chemical imaging correlated with the progress of mineral formation and weathering. Specific segments of mycorrhizal short roots were dissected from Pinus sp. seedlings collected in outdoor location, examined by light microscopy for presence of fungal hyphae, and processed for electron microscopy following a slightly modified protocol [2]. Due to the very delicate nature of the root / fungi associations, $1 \%$ gelatin was gently layered over the dissected samples to preserve the bacterial colonization of the mycorrhizosphere to physically withstand the processing during the plastic embedding for the TEM. SEM samples were intentionally left chemically unprocessed to avoid the possible material loss and chemical alteration of the minerals. Using a suite of state-ofthe-art imaging techniques such as high resolution transmission electron microscopy coupled with spectroscopy coupled with energy dispersive spectroscopy (Tecnai T-12 and Titan, FEI) and scanning electron microscopy and focused ion beam milling (Helios, FEI), we are able to relate the nanometer-scale alteration processes in the minerals with the physiology and the chemical micro-environment around the hyphae and at the interface with the mineral substrates. 
This unique combination of approaches will shed new light into the mechanism and rates of fungal weathering at the mineral surface, in particular the importance of the fungal hyphae in organic nutrient ( $\mathrm{N}$ and $\mathrm{P}$ ) mobilization, and plant-derived carbon translocation to the soils in the upcoming columns experiments.

[1] Balogh-Brunstad Z. et al. Biogeochemistry (88) 2, 153-167. (2008)

[2] Sarand I. et al. FEMS Microbiology Ecology (27) 115-126(1998)

[3] This research was performed at the W. R. Wiley Environmental Molecular Sciences Laboratory, a national scientific user facility sponsored by the U.S. DOE, located at PNNL. We would like to thank Dr. James Harsh (WSU) for helpful comments.
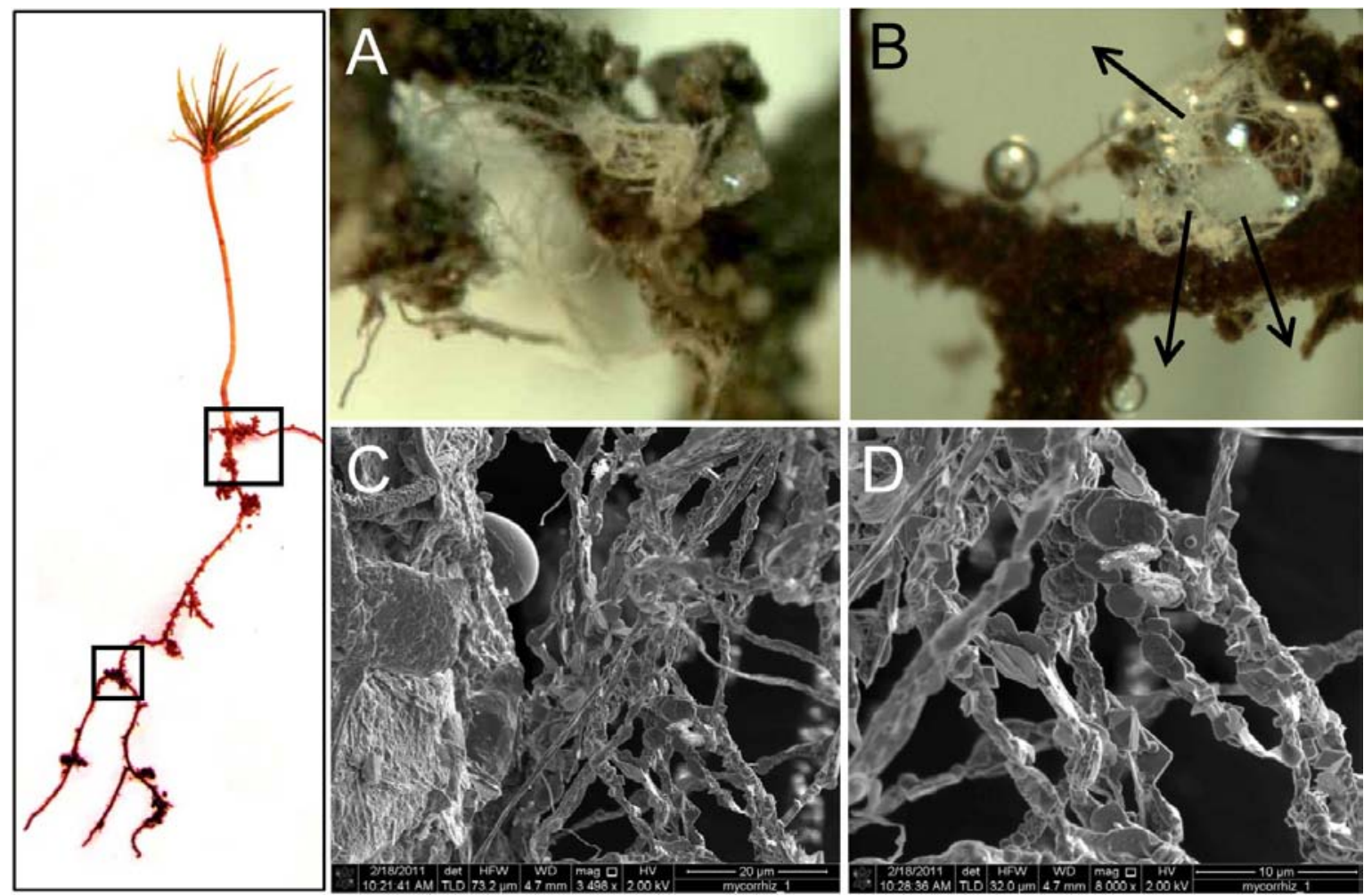

Fig.1. Root system of a Pinus sp. seedling with mycorrhizal short roots (inset), sites of mycorrhizal fungus colonization (A). Fine hyphae tend to widely disperse during the chemical fixation and ethanol dehydration (B); gelatin enrobing was used instead to avoid loss of material and to preserve the architecture. SEM images of fungal hyphae in the close proximity of a root surface (C), often covered by microcrystalline material (D). 